\title{
Contributions to a revision of Cercidospora (Dothideales), 2: Species on Lecanora s. 1., Rhizoplaca and Squamarina
}

\author{
Calatayud $\mathbf{V}^{1}$, Navarro-Rosinés $\mathbf{P}^{2}$ and Hafellner $\mathbf{J}^{3}$ \\ ${ }^{1}$ Fundación CEAM, Parc Tecnològic, Ch. R. Darwin 14, ES-46980 Paterna, València, Spain \\ ${ }^{2}$ Dept. de Biologia Vegetal (Botànica), Fac. de Biologia, Univ. de Barcelona, Av. Diagonal 645, ES-08028 Barcelona, \\ Spain \\ ${ }^{3}$ Institut für Pflanzenwissenschaften, Karl-Franzens-Universität, Holteigasse 6, A-8010 Graz, Austria
}

Calatayud V, Navarro-Rosinés P, Hafellner J 2013 - Contributions to a revision of Cercidospora (Dothideales), 2: Species on Lecanora s. 1., Rhizoplaca and Squamarina. Mycosphere 4(3), 539557, Doi 10.5943/mycosphere/4/3/8

\begin{abstract}
A study on the taxonomy, morphology and anatomy of the lichenicolous species of the genus Cercidospora (Dothideales, incertae sedis) growing on lichens of the genera Lecanora (Lecanoraceae), specifically of the L. polytropa group and the L. saxicola group (i.e. L. muralis sensu auct. group, Protoparmeliopsis spp.), Rhizoplaca (Lecanoraceae) and Squamarina (Stereocaulaceae) is presented. The following species are proposed as new: Cercidospora barrenoana on Rhizoplaca peltata, and C. melanophthalmae on Rhizoplaca melanophthalma. $C$. stenotropae is proposed provisionally; this fungus grows on Lecanora stenotropa and other taxa of the L. polytropa group. A key for the species of the genus Cercidospora treated is provided.
\end{abstract}

Key words - Ascomycota - lichenicolous fungi - lichenized fungi

\section{Introduction}

This study is the second contribution to the revision of the genus Cercidospora (Dothideales, incertae sedis) which is being undertaken. It is centered on the species growing on lichens of the genera Lecanora s. 1. (Lecanoraceae, Lecanorales), specifically of the L. polytropa group and $L$. saxicola group (i.e. L. muralis sensu auct. group, Protoparmeliopsis spp.), Rhizoplaca (Lecanoraceae, Lecanorales), and Squamarina (Stereocaulaceae, Lecanorales; fide Miadlikowska et al. 2006).

Of the six species here treated, three were previously known. Two species, Cercidospora epipolytropa and C. macrospora (=C. ulothii), have been profusely cited. The third, C. crozalsiana, is recently recovered (Navarro-Rosinés et al. 1995).

The general features of the genus Cercidospora have been described in detail in a first contribution for the revision of the genus (Navarro-Rosinés et al. 2009), as well as in the studies by Hafellner (1987), Grube \& Hafellner (1990) and Navarro-Rosinés et al. (2004).

\section{Material and methods}

For the microscopic study of the morphology and anatomy of the species, sections of ascomata were prepared by hand, and mounted in water or, to increase the contrast, in lactophenol- 
cotton blue. All measurements of the different structures were made in water. For the illustrations, a drawing tube fitted to the microscope was used. In the size of the ascospores, the values in italics indicate the average value of length and width, the values in brackets are the extreme values, and the remaining values are the extreme values after rejecting $10 \%$ of the highest and $10 \%$ of the lowest values. The nomenclature of the host species follows Clauzade \& Roux $(1985,1987,1989)$, Hafellner \& Türk (2001) and Nimis (1993), except for some commented exceptions.

\section{Key to the species of Cercidospora treated}

1. Ascospores mostly over $25 \mu \mathrm{m}$ long, from ellipsoid-fusiform to cylindrical; asci over $100 \mu \mathrm{m}$

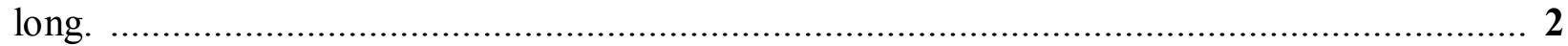

$\mathbf{1}^{*}$. Ascospores, usually less than $25 \mu \mathrm{m}$ long; asci not over $100 \mu \mathrm{m}$ long. .................................. 3

2(1). Ascospores c. 30-38 × 6-8 $\mu \mathrm{m}$; asci 90-145 × 10-15 $\mu \mathrm{m}$, 4-spored; ascomata 250-340 (400) $\mu \mathrm{m}$ diam; on Rhizoplaca peltata.

C. barrenoana

$2^{*}$. Ascospores somewhat smaller, c. $24.5-32 \times 5.5-7 \mu \mathrm{m}$; asci 85-120 × 10-14 $\mu \mathrm{m}$, with (2-)4 spores; ascomata c. 200-280 $\mu \mathrm{m}$ diam.; on Squamarina spp.

C. crozalsiana

$3\left(1^{*}\right)$. Asci mostly 8 -spored.

$\mathbf{3}^{*}$. Asci mostly 4-spored.

4(3). Ascospores c.18-22 × 5-6.5 $\mu \mathrm{m}$, ellipsoid or, mostly, fusiform; asci c. 70-90 × 10-15 $\mu \mathrm{m}$, with (6-)8 spores; ascomata 170-330 $\mu \mathrm{m}$ diam.; on Rhizoplaca melanophthalma.

C. melanophthalmae

4*. Ascospores somewhat smaller, c. 15-19 × 5-6 $\mu \mathrm{m}$, oval-ellipsoid; asci 50-70 9 9-13 $\mu \mathrm{m}$, with (4-6-)8 spores; ascomata 130-220 $\mu \mathrm{m}$ diam.; on Lecanora polytropa s. str., L. intricata and other taxa of $L$. polytropa group.

C. epipolytropa

$5\left(3^{*}\right)$. Ascospores c. 20-25 $\times 4-6 \mu \mathrm{m}$, narrowly ellipsoid or fusiform; asci c. $65-90 \times 9-11 \mu \mathrm{m}$, with $4(-8)$ spores; ascomata $150-250 \mu \mathrm{m}$ diam.; on Lecanora saxicola group (=Lecanora muralis sensu auct. group).

C. macrospora

$\mathbf{5}^{*}$. Ascospores c. $16-21 \times 5-6 \mu \mathrm{m}$, from narrowly ellipsoid to slightly fusiform; asci c. $40-55 \times 8-$ $10 \mu \mathrm{m}$, with (2-)4 spores; ascomata c. 110-150 $\mu \mathrm{m}$ diam.; on Lecanora stenotropa and other taxa of L. polytropa group (and possibly on Rhizoplaca aspidophora).

C. stenotropae

\section{The species}

Cercidospora barrenoana Calat. \& Nav.-Ros., sp. nov.

Fig. 1-9 a-e

MycoBank 516548

Ascomata perithecioidea, totaliter immersa in thallis hospitis. In sectione transversali pseudothecia globosa, 250-340 (-400) $\mu \mathrm{m}$ in diametro. Paries ascomatum apicaliter violascens, parce incrassatus, basaliter subhyalini-violascens, 30-40 $\mu \mathrm{m}$ crassus. Paraphysoides copiosae, (1.52(-3) $\mu \mathrm{m}$ in diametro. Asci cylindrici, circa 90-145 $\mu \mathrm{m}$ longi et 10-15 $\mu \mathrm{m}$ lati, tetraspori. Ascosporae (27-)30-38(-40) $\times(5-) 6-8 \mu \mathrm{m}$ magnae, incoloratae, longe ellipsoideo-fusiformes vel (sub)cylindriceae, ad septum non aut parum constrictae, cellula superiore breviore crassioreque quam cellula inferior, tenuiter halonatae. Cercidosporae macrosporae et $C$. crozalsianae affinis, sed ab eis dissimilis pseudotheciis et ascosporis majoribus. Supra thallum Rhizoplacae peltatae vigens.

Type - Spain: Islas Canarias, Tenerife, Parque Nacional de El Teide, Roques de García, rocas volcánicas, 2125 m, 23.IX.1993, V. Calatayud (VAB-lich. 7436, holotype).

Host species of the type - Rhizoplaca peltata (Ramond) Leuckert \& Poelt. 

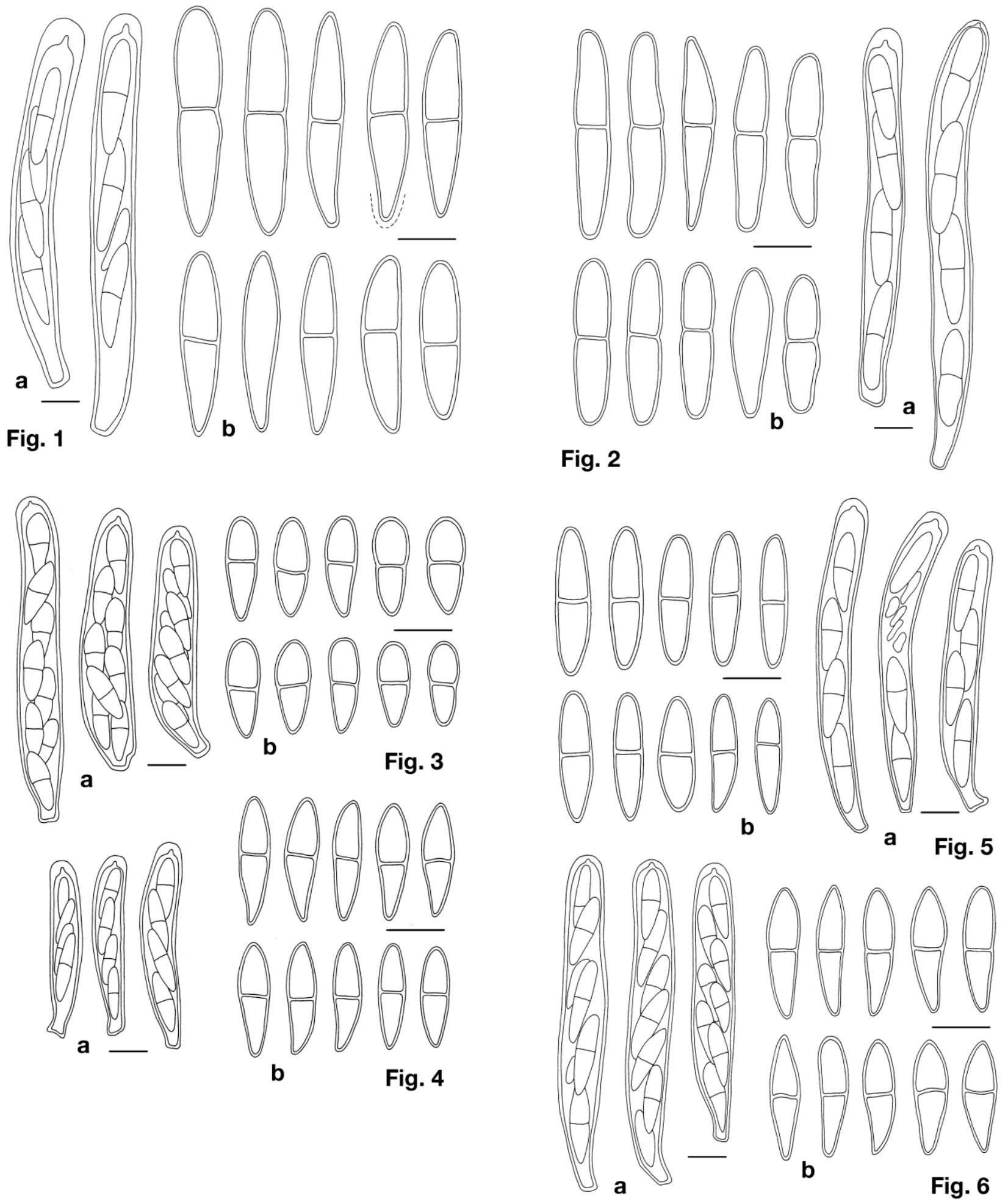

Fig. 1 -Cercidospora barrenoana (holotype). Fig. 2 -Cercidospora crozalsiana (Gordes, Vaucluse, France; BCN 13301-13305). Fig. 3-Cercidospora epipolytropa (Anielarra, Aragón, Spain; BCN-lich. 2549). Fig. 4 - Cercidospora stenotropae (Meranges, Catalonia, Spain). Fig. 5 Cercidospora macrospora (Escatron, Aragón, Spain). Fig. 6-Cercidospora melanophthalmae (Col de Lautaret, Alpes sept. du Dauphinée, France; MARSSJ, Lichenes Alpium 49). In all figures: a, asci; b, ascospores. - Bars $=10 \mu \mathrm{m}$. 
Etymology - barrenoana (Lat.), belonging to Barreno; named after Dr. Eva Barreno (professor of Universitat de València).

Description - Ascomata perithecioid, 250-340(-400) $\mu \mathrm{m}$ diam., provided with a violaceous exciple, rarely with a greenish tinge in its upper part, less pigmented towards its base; in mature ascomata sometimes uniformly violaceous-colored throughout and with a greenish tinge, 30-40 $\mu \mathrm{m}$ thick towards the base. Paraphysoids abundant, (1.5-)2(-3) $\mu \mathrm{m}$ thick. Asci $90-145 \times 10-15 \mu \mathrm{m}$, cylindrical, 4-spored. Ascospores (27-)30-33.4-38(-40) $\times(5-) 6-6.8-8 \mu \mathrm{m}$, with a length/width ratio of $(3.6-) 4.3-5.0-5.8(-7.2)(n=59)$, mostly 1 -septate, but simple ascospores also abundant, colorless, narrowly ellipsoid-fusiform to almost cylindrical, slightly heteropolar, not or only slightly constricted at the septum, mostly with a clearly visible, gelatinous sheath, $1-2 \mu \mathrm{m}$ thick, guttulate.

Remarks - Cercidospora barrenoana and C. crozalsiana are the two species in this study showing the largest asci and ascospores, and clearly exceeding the sizes of the structures of the rest of the taxa. C. barrenoana is the species of the genus with the largest ascospores, with a length of mostly 30-40 $\mu \mathrm{m}$. In $C$. crozalsiana, the ascospores are slightly smaller, with a predominant length of $24-32 \mu \mathrm{m}$, and only occasionally with ascospores reaching $37 \mu \mathrm{m}$ long. The ascomata of $C$. barrenoana, which are usually $250-340 \mu \mathrm{m}$ in diam., but can reach $400 \mu \mathrm{m}$ in some cases, is among the largest in the genus. In most of the Cercidospora species the diam. of the ascomata is clearly smaller. Only in C. melanophthalmae (see below) and C. mutabilicola sp. ined. (NavarroRosinés et al. 2009), can they also be over $300 \mu \mathrm{m}$ wide.

Distribution and habitat - To date, C. barrenoana is known only from its type locality in Tenerife (Canary Islands), growing on Rhizoplaca peltata as a parasymbiont. Halıc \& Aksoy (2009) cite Cercidospora ulothii upon Rhizoplaca peltata from Turkey, a specimen that might also belong here. A thorough screening of the material of $R$. peltata stored in the herbarium GZU did not result in additional findings.

Cercidospora crozalsiana (H. Olivier) Nav.-Ros., Cl. Roux \& Casares.

Fig. 2

Cryptogamie, Bryol. Lichénol. 16(2): 100 (1995).

三Sphaeria crozalsiana H. Olivier, Bull. Acad. Internat. Géogr. Bot. 17: 168 (1907).

三Didymella crozalsiana (H. Olivier) Vouaux, Bull. Soc. Mycol. France 29: 98 (1913).

Type - [France], "Hérault, à Béziers, sur le Squamaria lentigera, [A.] de Crozals“ (PC?, n.v.).

Host species of the type - Squamarina lentigera (Weber) Poelt.

Description - Ascomata perithecioid, (160-)200-280 $\mu \mathrm{m}$ diam., exciple blue-green in its upper part and colorless at the base; in the most mature ones sometimes uniformly blue-green throughout, $12-15(-20) \mu \mathrm{m}$ thick towards its base. Paraphysoids abundant, $1.5-2 \mu \mathrm{m}$ thick. Asci $85-120 \times 10-14 \mu \mathrm{m}$, cylindrical, mostly 4-spored, but not rarely 2 -spored. Ascospores (22-)24.5$28.3-32(-37) \times(5-) 5.5-6.2-7(-8) \mu \mathrm{m}$, with a length/width ratio of $(3.2-) 3.7-4.6-5.5(-6.8)$ ( $n=106)$, mostly 1-septate, only occasionally simple, colorless, from narrowly ellipsoid-fusiform to almost cylindrical, slightly heteropolar, not or only slightly constricted at the septum, mostly with a clearly visible gelatinous sheath, guttulate. Pycnidiospores bacilliform, 3-5(-8) $\times 0.5-1 \mu \mathrm{m}$.

Remarks - Cercidospora crozalsiana is close to C. macrospora and C. barrenoana in having 4-spored asci and in the shape and size of the ascospores. However, $C$. crozalsiana differs from the two mentioned species in the intermediate size of its ascospores, larger than those of $C$. macrospora (mostly 20-25 $\mu \mathrm{m}$ long), but smaller than those of $C$. barrenoana (mostly 30-40 $\mu \mathrm{m}$ long). The asci in $C$. crozalsiana, 85-120 $\mu \mathrm{m}$ long, are also among the largest in the genus; this length is only exceeded in the case of $C$. barrenoana, with asci 90-140 $\mu \mathrm{m}$ long. In $C$. macrospora, they are only 65-90 $\mu \mathrm{m}$ long. Comments on the nomenclature and the different criteria applied by several authors for this species are given in Navarro-Rosinés et al. (1995).

Distribution and habitat - Cercidospora crozalsiana is known from several Mediterranean localities in the Iberian Peninsula and southern France, mentioned in Navarro-Rosinés et al. (1995), and more recently it has been also reported from Turkey, by Candal \& Halıc1 (2011). Furthermore we report it here from southern Sweden and the Balcan country Macedonia. Based on our 
observations, this species is regarded as a specific parasymbiont of Squamarina species, among which we could identify $S$. cartilaginea, S. lentigera and S. stella-petraea. The two former host species grew on soil, including gypsum-rich soils, while the latter grew on boulders of carbonated sandstone, close to the soil.

Exsiccata examined - Hafellner, Lichenicolous Biota 14 (BR, CANB, GZU, NY, UPS).

Specimens examined - EUROPE: France: See Navarro-Rosinés et al. (1995).- Macedonia: Scopje, secus viam inter Želino et Grupčin, 280 m, ad saxa calcarea, 6.X.1976, A. Vězda (GZU). On Squamarina cartilaginea.- Korab, Tal der Radika bei Kosovrasti Banja E von Debar, SEexponierte Hänge aus Gips über der Schwefelquelle; 10.VII.1977, J. Hafellner 52501 (herb. Hafellner). On S. lentigera.- Spain: See also Navarro-Rosinés et al. (1995) and Hafellner \& Casares-Porcel (2003).- Aragón, Zaragoza, entre Fuentejalón y Pozuelo de Aragón, lomas yesosas cerca de la carretera, $450 \mathrm{~m}$, 4.IV.1999, J. Etayo (herb. J. Etayo 16970, BCN-lich.). On $S$. lentigera.- Sweden: Öland, between Sandviken and Södvik, on alvar ground (calcareous soil), 12.VII.1977, I. Kärnefelt 2710a (GZU). On S. lentigera.

Cercidospora epipolytropa (Mudd) Arnold.

Fig. 3-7 k-p

Flora 57:154 (1874).

三 Thelidium epipolytropum Mudd, Man. Brit. Lichens: 298 (1861).

$\equiv$ Verrucaria epipolytropa (Mudd) Crombie, Lich. Brit.: 121 (1870).

三 Pharcidia epipolytropa (Mudd) Arnold, Flora 53: 236 (1870).

三 Didymosphaeria epipolytropa (Mudd) G. Winter, Rabenh. Krypt.-Fl., Ed. 2, 1(2): 432 (1885).

三Didymella epipolytropa (Mudd) Berl. \& Voglino, Syll. Fung. Add. 1-4: 89 (1886).

三 Cyrtidula epipolytropa (Mudd) Jatta, Syll. Lich. Ital.: 496 (1900).

三Arthopyrenia epipolytropa (Mudd) H. Olivier, Bull. Int. Acad. Géogr. Bot. 16: 262 (1906).

Type - British Isles: sine loc., on Lecanora polytropa, W. Mudd (K 163703, neotype ("lectotypus") selected by Hawksworth \& Diederich 1988).

Loci classici of syntypes - Great Britain, Highlands of Scotland, Admiral Jones; Ayton Moor, Cleveland, W. Mudd (?), both on Lecanora polytropa. Cliffrigg near Ayton, Cleveland, W. Mudd (?), on Lecanora saxicola as Squamaria saxicola.

Host species of the type - Lecanora polytropa (Ehrh. in Hoffm.) Rabenh.

Illustrations - Hafellner (1987: 358).

Description - Ascomata preferably immersed in the hymenia of the host, perithecioid, (100-) 130-220 $\mu \mathrm{m}$ diam., globose. Exciple colorless in its lower half, greenish or \pm brownish blue around the ostiole, $10-15 \mu \mathrm{m}$ thick toward its base. Paraphysoids abundant, 1-1,5 $\mu \mathrm{m}$ thick, only scarcely branched. Asci 50-70 × 9-13 $\mu \mathrm{m}$, cylindrical-clavate, mostly 8-spored, rarely only 4- or 6-spored. Ascospores (14-)15-17.3-19(-22) $\times(4.5-) 5-5.5-6(-7) \mu \mathrm{m}$, with a length/width ratio of (2.4-)2.73.1-3.8 $(-4.2)(n=99)$, 1-septate, very rarely with some simple or 3-septate spores, oval-ellipsoid to slightly fusiform, with both cells similar in size and a median septum, but slightly heteropolar, with the lower cell slightly narrower than the upper one. Pycnidia globose, 100-130 $\mu \mathrm{m}$. Pycnidiospores colorless, simple, bacilliform, 3-4(-5) $\times 0.5-1 \mu \mathrm{m}$.

Remarks - Cercidospora epipolytropa s. l. is a fungus specific to different taxa of the Lecanora polytropa group, and is characterized by having ascospores varying from oval-ellipsoid to fusiform, mostly below $20 \mu \mathrm{m}$ in length. This size is coincident with the values given by other authors on the same hosts (Hafellner 1987, Vainio 1921).

Setting aside the size of the ascospores, there are a number of constant differences among the different specimens of $C$. epipolytropa s. 1. studied. The most striking one is the number of ascospores per ascus, 4 or 8 , as the case may be; this difference seems to be associated with variations in the size of asci and ascomata, and also with host characteristics. 8-spored specimens grow mainly on typical Lecanora polytropa, proceeding from localities at relatively high altitudes. 
On the contrary, the samples of Cercidospora with 4-spored asci come from localities at lower altitudes, and grow mostly on a taxon of the L. polytropa group which fits the features of $L$. stenotropa Nyl. (according to Clauzade \& Roux 1985, Purvis et al. 1992, Wirth 1995).

In this study, the specimens with 4-spored asci are separated into a provisional species, Cercidospora stenotropae, pending the availability of more Cercidospora material growing on the L. polytropa group. The examination of more material would allow us to determine the characteristics and variability of these fungi more exactly, and have a better knowledge of their distribution, and clearer differentiation of their hosts.

In the original description of Thelidium epipolytropum, Mudd (1861) applied a wider concept of this taxon which would include both Cercidospora epipolytropa s. str. and C. macrospora as currently delimited as he reported specimens growing on Lecanora polytropa and on Lecanora saxicola (sub Squamaria saxicola). Specimens growing on L. saxicola and possibly constituting paratype material were distributed in an exsiccatum by itself (Mudd, Lich. Brit. Exs. 287). A sample of it was revised as Cercidospora macrospora (see below). The neotype ("lectotypus") of Thelidium epipolytropum was selected by Hawksworth \& Diederich (1988) from a specimen on Lecanora polytropa collected by W. Mudd from an unknown locality. This type material is characterized by having 8-spored asci and other features fitting our concept of $C$. epipolytropa s. str.

There are also reports of $C$. epipolytropa upon the thallus of Lecanora geophila, a terricolous species with e secondary chemistry similar to that of Lecanora polytropa. Such material originating from western Siberia has been distributed by Santesson, Fungi Lichenicoli exs. 257, accompanied by a note on the label that in this strain 4-spored and 8-spored asci should be equally frequent and the ascospores being 16-18 $\times 5-7 \mu \mathrm{m}$ (Santesson 1998). The strain has also been reported from Greenland inhabiting even the type specimen of the host lichen (Obermayer \& Kantvilas 2003). It needs to be further studied as are other records of $C$. epipolytropa on single specimens of Lecanora baicalensis and L. chondroderma (Poelt \& Grube 1993).

Furthermore, $C$. epipolytropa has also been mentioned as a parasite of Rhizoplaca melanophthalma (Navarro-Rosinés \& Hladun 1987, Alstrup \& Hawksworth 1990). However, the specimens growing on this host differ from the ones associated with Lecanora polytropa group by having larger ascomata and slightly longer, ellipsoid-fusiform ascospores. In this work, they are separated into a new species: $C$. melanophthalmae.

Distribution and habitat - Cercidospora epipolytropa is a taxon widely distributed in the Northern Hemisphere, profusely cited in Europe, North America (Hafellner 1987, Triebel et al. 1991). Records from Asia (apart from Siberia, see Zhurbenko 2007) and Africa are still few. After our observations, this species may be regarded as a parasymbiont (although its ascomata are also developed in the hymenia of the host lichens) of Lecanora polytropa s. str., L. polytropa var. alpigena, L. intricata and other taxa of this group dwelling in northern territories and in high mountains. In fact, C. epipolytropa is the lichenicolous fungus holding the altitude record on earth. It has been reported together with its typical host from Makalu in the Himalaya range at $7400 \mathrm{~m} \mathrm{~s}$. m. (Hafellner 1987).

Exsiccata examined - Hafellner, Lichenicolous Biota 32 (BR, CANB, GZU, NY, UPS).Räsänen, Lichenes Fenniae Exs. 700 (GZU).- Santesson, Fungi Lichenicoli Exs. 157 (GZU and herb. P. Diederich).

Selected specimens examined - (on Lecanora polytropa if not otherwise stated).

EuroPE: Austria: Kärnten, Hohe Tauern, Kreuzeck-Gruppe, Hänge oberhalb der Turgger Alm gegen die Schwarzsteinwände, Grünschiefer, 46²4'N/1309' E, 2000-2100 m, GF 9244/2, 24.VII.1994, J. Poelt (GZU).- Kärnten, Klagenfurter Becken, Kreuzberg bei Klagenfurt, IX.1973, J. Poelt (GZU).- Niederösterreich, Waldviertel, große Granitfelsen ca. 1,4 km W Pretrobruck, ca. 820 m, GF 7555/2, 7.IV.1994, J. Poelt \& R. Türk (GZU).- Steiermark, Schladminger Tauern, NWAbhänge des Säulecks im Sattental, S von Gröbming, am oberen Ende des Schneetalrückens, Schieferschrofen, ca. 2150 m, 3.IV.1985, J. Hafellner 13025 (GZU).- Steiermark, Niedere Tauern,Wölzr Tauern, Eselsberggraben NW von Oberwölz, ca. 1 km SE der Neunkirchner Hütte, 

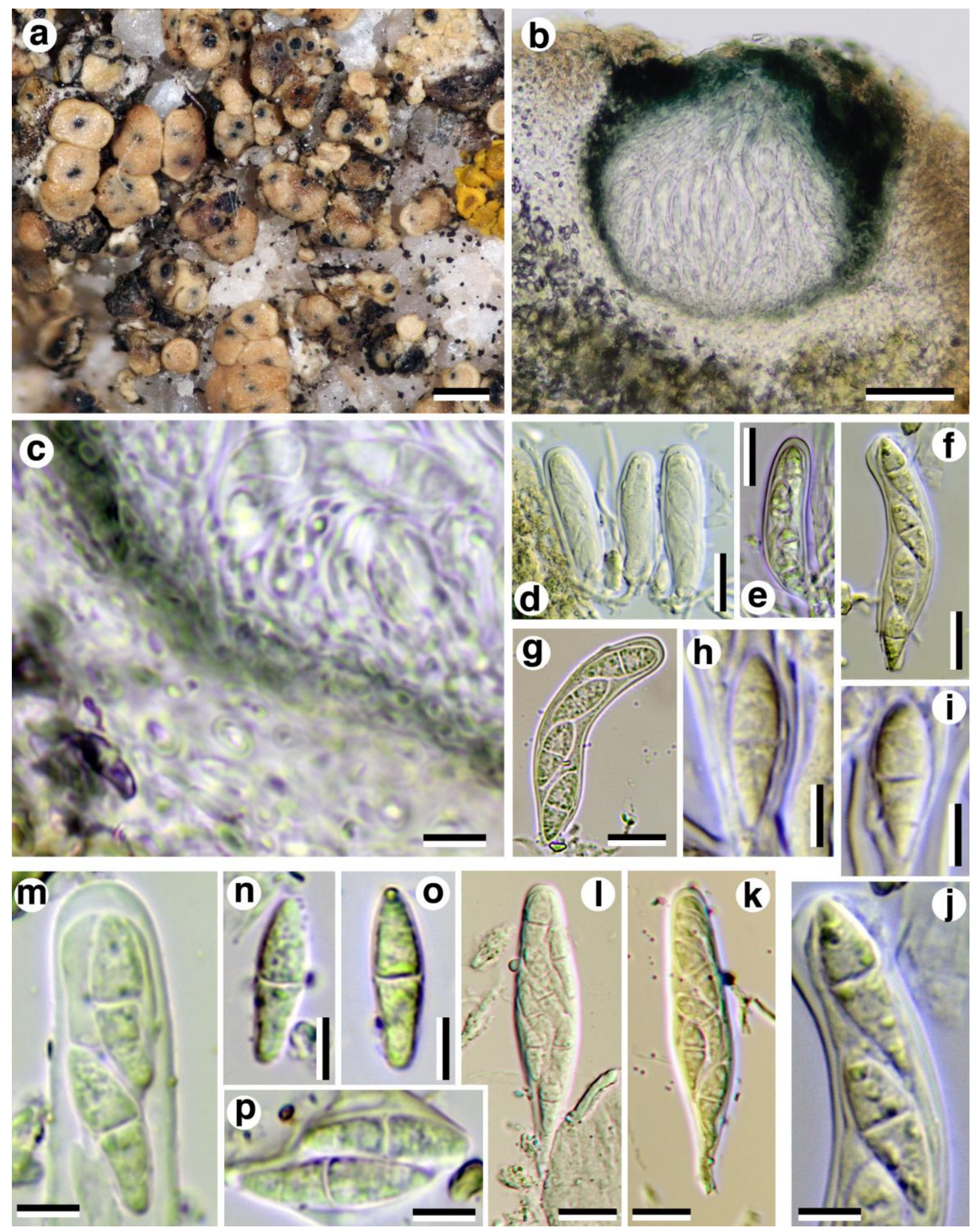

Fig. 7 - a-j Cercidospora stenotropa (Soria, Spain, VAB 7726). a superficial aspect of a parasitized thallus of Lecanora gr. polytropa, with the fungal ascomata immersed in the lichen apothecia. b detail of the ascoma in vertical section. $\mathrm{c}$ detail of the exciple in vertical section from the basal part of the ascoma. $\mathrm{d}$-e immature asci $\mathrm{f}-\mathrm{g}$ mature asci. $\mathrm{h}-\mathrm{j}$ ascospores. $\mathrm{k}-\mathrm{p}$ Cercidospora epipolytropa (Apache, Arizona, USA, ASU-Nash 11763). k-1 immature asci. $\mathrm{m}-\mathrm{p}$ ascospores. - Bars: $\mathrm{a}=0.2 \mathrm{~mm} ; \mathrm{b}=50 \mu \mathrm{m} ; \mathrm{d}-\mathrm{g}, \mathrm{k}-\mathrm{l}=10 \mu \mathrm{m} ; \mathrm{h}-\mathrm{j} \mathrm{m}-\mathrm{p}=5 \mu \mathrm{m}$.

[47 $16^{\prime} 15^{\prime}$ 'N $/ 14^{\circ} 09^{\prime} 30^{\prime}$ 'E], ca. 1510 m, GF 8750/2, teilweise eisenhältige Glimmerschieferblöcke, 22.V.1988, J. Hafellner 20850 \& A. Hafellner (GZU).- Steiermark, Seetaler Alpen, W- Abhang 5 km E von Neumarkt, Oberberg, 200 m unter dem Gehöft Schweintaler, 1250 m (GF 8952/2), offene Waldweide, Felsblöcke, 20.III. 1987, W. Obermayer 1934 (GZU). On Lecanora intricata.- 
Steiermark, Joglland, N Schloss Herberstein, unterste N- und W-seitige Abbrüche des Buchberges gegen den Stubenbergsee bzw. die Feistritz, 400-420 m, 6.XI.1988, J. Poelt (GZU).- Steiermark, Steirisches Randgebirge („Cetische Alpen“), Wechsel, an der Bergstraße von Mönichwald zum Wechsel, am Wiesenhang oberhalb vom Spitzbauer, ca. 1000 m, GF 8561, Lesesteinhaufen, 8.IX.1989, J. Poelt (GZU).- Tirol, Ötztaler Alpen, Ötztal, 3 km SSE von Obergurgl, E der Brücke über den Gaisbergtal-Bach, 2300-2400 m, 30.VIII.1993, J. Poelt (GZU). On L. polytropa var. alpigena.- Tirol, Osttirol, Nationalpark Hohe Tauern, Glockner-Gruppe, Ködnitztal NE ober Kals,

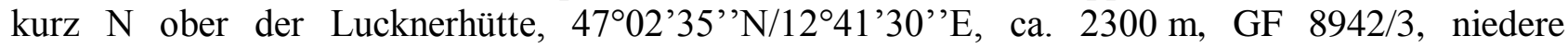
Kalkschieferschrofen und Rasen am Westhang, Lesesteinmauer, auf Glimmerschiefer, 4.IX.1998, J. Hafellner 46861 (GZU).- See also Hafellner (1987).- France: Dept. Puy-de-Dôme: Auvergne, monts Dore, puy de Sancy, Andesitschrofen auf der Nordseite, ca. 1820 m, 31.VII.1980, A. Bellemère \& J. Hafellner 9550 (GZU).- Dept. Cantal: Auvergne, monts du Cantal, puy Mary, am Steig vom Pas de Peyrol zum Gipfel, ca. 1680 m; NW-exponierte Andesitblöcke, 30.VII.1980, A. Bellemère \& J. Hafellner 9421 (GZU).- See also Hafellner (1987).- Germany: See Hafellner (1987).- Italy: Südtirol, Zentralalpen, Ötztaler Alpen, Weißseejoch N von Melag im Langtauferertal, [46'52'00' N/1041'25',E], 2960 m, Schiefer, 21.IV.1984, J. Hafellner 12455 (GZU).- See also Hafellner (1987).- Norway: Hordaland, Gem. Voss, S-exp. Hänge N des Sees Hamlagrövatnet, ca. $25 \mathrm{~km} \mathrm{SW}$ von Voss, ca. $800 \mathrm{~m}$, niedere Felshöcker aus Schiefer an der Obergrenze des Birkenwaldes, 20.VIII.1984, J. Hafellner 12107, 12190 \& A. Ochsenhofer (GZU).See also Hafellner (1987).- Slovakia: Niedere Tatra, oberhalb Špania Dolina, 740-770 m, Abraumhalde der alten Kupfermine, 1.VII.1993, I. Pišút \& J. Poelt (GZU, under L. polytropa).Spain: Aragón, prov. Huesca, Anielarra, Campa de la Contienda, 1700 m, 19.VII.1973, X. Llimona (BCN-lich 2546, in L. polytropa).- See also Hafellner (1987).- Sweden: Vätergötland, par. Partille to the north, on boulder, 23.V.1919, H. Magnusson, Swedish Lichens n. 2469 (MARSSJ).- See also Hafellner (1987).- Switzerland: See Hafellner (1987).- United Kingdom: Isle of Skye, S Carbost, Glen Brittle Hut, NG 41 21, on rocks, 28.V.1987, P. Diederich 9109 (herb. P. Diederich).- Isle of Skye, NE Uig, Quiraing, NG 44 68, on rocks, 24.V.1987, P. Diederich 8204 (herb. P. Diederich).

ASIA: Japan, Hokkaido, Prov. Ishikari: beside the trail between Bohgakudai and Fukiage

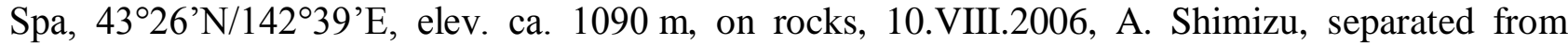
Kashiwadani, Lich. Minus Cogniti exs. 333) (GZU).- Nepal: See Hafellner (1987).

AFRICA: Canary Islands, Tenerife, an der Straße von La Orotava nach El Portillo, kurz unter El Portillo, [28 $18^{\prime} 40^{\prime}$ 'N $/ 16^{\circ} 34^{\prime} \mathrm{W}$ ], ca. $1950 \mathrm{~m}$, N-exponierte Vulkanitschrofen im lichten Pinus canariensis-Wald, 17.II.1989, J. Hafellner 32884 \& A. Hafellner (GZU).

NORTH AMERICA: U.S.A: Arizona, Apache Co., Escudilla Mountain, north of Alpine, sprucefir community, on volcanic rock, 10.500', 30.VIII.1975, T.H. Nash 11763 (ASU).- Arizona, Apache Co., Mount Baldy Wilderness, near the beginning of Trail No. 95, from Phelp's Cabin to

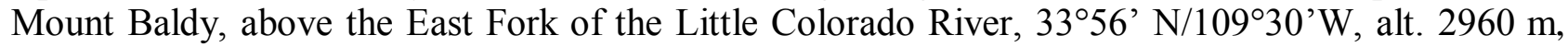
spruce-fir forest, on acidic rock, 9.VI.1998, T. H. Nash III $41983=$ Lich. exs. Arizona State Univ. 317 (GZU, under the name of the host).- California, Tulare, Sierra Nevada, Sequoia National Park, SE of Giant Forest, Mineral King, Eagle Crest Trail, NE exposed schist outcrops, with mature Juniperus, 2383 m, $118^{\circ} 35.7^{\prime} \mathrm{W} ; 36^{\circ} 26.9^{\prime} \mathrm{N}$, P. \& B. v.d.Boom (herb. Boom 29040).

Cercidospora macrospora (Uloth) Hafellner \& Nav.-Ros.,

Fig. 5-8

Lichen Flora of the Greater Sonoran Desert Region, Volume 2: 638 (2004).

三 Phacopsis macrospora Uloth, Flora 44: 653 (1861).

Type - [Germany], „parasitisch auf dem Laub von Placodium saxicolum auf Ziegeldächern auf den Gradirbauten bei Nauheim“, W. Uloth (?, n. v.).

= Cercidospora ulothii Körb., Parerga lich.: 466 (1865).

= Didymella ulothii (Körb.) Berl. \& Voglino, Syll. Fung. Add. 1-4: 89 (1886).

Type - [Germany], "auf dem Thallus von Placodium saxicolum,... bei Nauheim in Kurhessen”, [W.] Uloth (?, n. v.). 
? = Sphaeria crozalsiana var. saxicolae H. Olivier, Princip. Paras. Lich., Suppl. 1: 15 (1907).

Type - France, "Sur Squamaria saxicola à Roquehaute dans l'Hérault", De Crozals (?, n. v.).

Host species of the types - Lecanora saxicola (Pollich) Ach. (syn. Lecanora muralis sensu auct., Protoparmeliopsis muralis sensu auct.).

Illustrations - Hafellner (1987: 356), as C. ulothii.

Description - Lichenicolous fungus with the ascomata immersed in the host thallus and apothecial discs, producing, in some cases, deformations in the form of convex cecidia (observed in some specimens of Lecanora versicolor). Ascomata perithecioid, 150-250 mm diam. Exciple colorless or slightly green-blue in its lower half, intensely green-blue around the ostiole, 10-15(20) $\mu \mathrm{m}$ thick towards its base. Paraphysoids scarcely abundant, $1.5(-2) \mu \mathrm{m}$ thick. Asci $(55-) 65-$ $90 \times 9-11 \mu \mathrm{m}$, cylindrical, 4-spored, exceptionally 8-spored. Ascospores (19-)20-22.9-25(-30) $\times$ $4-5.1-6(-7) \mu \mathrm{m}$, with a length/width ratio of (3.0-)3.8-4.5-5.4(-6.7) $(n=179)$, 1-septate, narrowly ellipsoid or fusiform, with both cells equal in shape and size, with the septum median, more rarely slightly heteropolar.

Remarks - Cercidospora macrospora is a species characterized by having predominantly 4spored asci and ascospores with a length mostly of 20-25 $\mu \mathrm{m}$. Similar 4-spored asci also occur in $C$. barrenoana and $C$. crozalsiana, but in these species the size of the ascospores is clearly larger (see also the comments on these species). C. stenotropae also has 4-spored asci, but its ascospores measure only 16-21 $\mu \mathrm{m}$ in length, being smaller than those of $C$. macrospora.

For the synonymy of $C$. ulothii in $C$. macrospora see Navarro-Rosinés et al. (2004). According to Santesson (1960), both taxa were described on the basis of material likely to come from the same collection. Taking this into account and also considering that they were described on the same host, there is little doubt about their synonymy. Hence, the second name has priority over the well-known former one.

Other than Cercidospora epipolytropa, C. macrospora does not show a preference to develop the ascomata in the apothecial discs. Ascomata are equally frequent in host thallus and apothecia.

Distribution and habitat-Cercidospora macrospora is considered a holarctic taxon (Hafellner 1987). Previously this species has been reported most frequently under its synonym, $C$. ulothii. Most records are from Europe, temperate Asia, the Mediterranean and Macaronesian regions of Africa, and North America. Typically, it is associated with taxa of Lecanora saxicola group (i. e., the Lecanora muralis sensu auct. group or Protoparmeliopsis spp.), growing both on siliceous and calcareous substrata. Hosts that we have identified for $C$. macrospora include Lecanora bolcana, L. dubyi (= L. muralis subsp. dubyi), L. garovaglii, L. cf. nevadensis, L. novomexicana, L. phaedrophthalma var. christoi, L. saxicola (= L. muralis sensu auct., fide Laundon 2010) and L. versicolor.

Arup \& Grube (1999) and Pérez-Ortega et al. (2007) confirmed phylogenetically that Lecanora garovaglii is sister to Lecanora saxicola, although the former find L. novomexicana and L. phaedrophthalma more closely related to Rhizoplaca melanophthalma than to L. saxicola. It would be necessary to confirm these hosts, with the study of more specimens of $C$. macrospora on L. novomexicana and L. phaedrophthalma.

Although relatively rare, C. macrospora can be locally abundant, as in locality of Aragón cited below where it covered important extensions of the thalli of $L$. versicolor growing on horizontal carbonated sandstone surfaces. Citations of C. macrospora (or C. ulothii) on hosts other than the L. saxicola group most likely belong to other taxa (see also under $C$. barrenoana and $C$. melanophthalmae).

Exsiccata examined - Hafellner, Lichenicolous Biota 134 (BR, CANB, GZU, NY, UPS).Kunze, Fungi Selecti Exs. 78 (GZU).- Santesson, Fungi Lichenicoli Exs. 203 (GZU), 258 (GZU and herb. P. Diederich).- Triebel, Microfungi Exs. 181 (GZU).

Selected specimens examined - (on Lecanora saxicola var. saxicola unless otherwise cited). 
EUROPE: Albania, Northern Albania, Shkodër distr., Shkodër, Rozafa (hill of the castle) 42 $02^{\prime} 30^{\prime \prime} \mathrm{N} / 19^{\circ} 29^{\prime} 30^{\prime}$ 'E, c. $100 \mathrm{~m}$; limestone, on rock outcrops, 11.IV.2001, L. Kashta, herb. Hafellner 54902 (GZU).- Austria: Kärnten, Nationalpark Hohe Tauern, Glockner-Gruppe, S

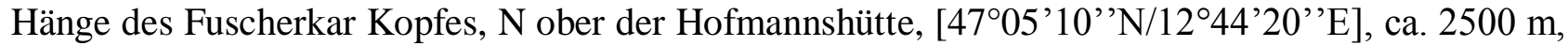
GF 8942/1, Kieselkalkblöcke, 21.IX.1988, J. Hafellner 32052, M. Walther \& A. Hafellner (herb. Hafellner). On L. dubyi.- Steiermark, Niedere Tauern, Triebener Tauern S von Trieben, Großer

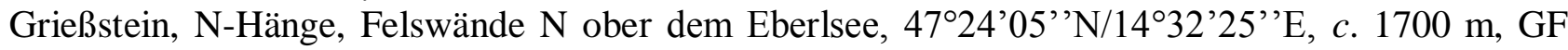
8553/3, SE-exponierte Felsabbrüche eines quarzreichen Silikatgesteins mit Spuren von Karbonat, auf Steilflächen am Fuß der Abbrüche, 15.VIII.2000, J. Hafellner 52170 (GZU).- Tirol, Paznaun, Fimbatal, nahe dem Ghf Bodenalpe, IX.1960, A. Schröppel (GZU). On L. dubyi.- See also Hafellner (1987).- France, Dept. Hautes-Alpes (05), entre le Lautaret et le Galibier, versant S de la crête de Chailloll, $2320 \mathrm{~m}$, sur schistes à peine calcaires, $80^{\circ} \mathrm{S}$, in Acarosporetum badiofuscae, 28.VIII.1972, C. Roux (MARSSJ). On L. saxicola agg.- Dept. Hautes-Alpes, col de Granon, NNW von Briancon, 2250-2400 m, Gneisfelsen, 11.VII.1970, J. Poelt 8571 (GZU, under the name of the host). On L. dubyi.- Germany, Reinland, Eifel, Schotterhang zwischen Mechernich und Dattel, VIII.1978, B. Feige (GZU).- Sachsen-Anhalt, W von Eisleben, Kupferschieferhalde zwischen F 80 und Saugrund, 11.XI.1990, S. Huneck (GZU).- Greece: Makedonien, Vermion Gebirge, zwischen Veria und Kozani oberhalb der Quelle Zoodochon pighi, alt. 1500 m, 22.VII.1973, H. Pittoni (GZU).- Voiotia, Bergrücken SE der Ortschaft Tsoukalades, N der Straße von Livadia nach Arahova, alt. ca. 500 m, 16.IX.1989, H. Mayrhofer 16314 (GZU). On L. dubyi-- Italy: TrentinoAlto Adige, Südtirol/prov. Bolzano, Vinschgau, Trockenhang bei Laas, alt. ca. 870 m, 10.X.1953, A.Schröppel (GZU). On Lecanora garovaglii.- Trentino, Südtiroler Dolomiten, Porto Vescovo S von Arabba, Felszacken kurz E über der Bergstation der Seilbahn, ca. $2400 \mathrm{~m}, 46^{\circ} 28^{\prime} \mathrm{N} / 11^{\circ} 53^{\prime} \mathrm{E}$, Sexponierte Abbrüche, 16.IV.1979, J. Hafellner 4595 (herb. Hafellner). On L. dubyi.- Trentino, Val di Fiemme, beim Rif. Salanzada S von Cavalese, [46 $16^{\prime} 30^{\prime}$ 'N $/ 11^{\circ} 27^{\prime} 15^{\prime \prime} \mathrm{E}$ ], ca. $1050 \mathrm{~m}$; Sexponierte Silikatschrofen; 27.X.1984, J. Hafellner 11891 (herb. Hafellner). On L. garovaglii.Emilia-Romagna, Prov. Parma, nördlicher Apennin, an der Straße über den [ca. $13 \mathrm{~km} \mathrm{NE} \mathrm{vom]}$ Pso. della Cisa, Mte. Prinzera am N Ortsende von Boschi di Bardone, [44³3'20'N/1002'10' 'E], ca. $850 \mathrm{~m}$, Serpentinit, SW-exponierte Abbrüche, 29.X.1978, J. Hafellner 4380 (GZU). On L.

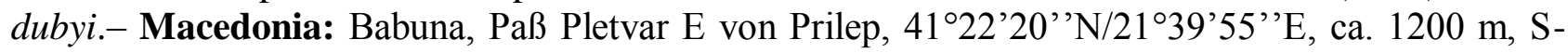
exponierte Hänge mit einzelnen großen Silikatblöcken, 11.VII.1977, J. Hafellner 41727 (herb. Hafellner). On L. garovaglii.- See also Hafellner (1987).- Netherlands (Limburg), Maastricht, stadsmuur bij de hoek van de Nieuwenhofstraat, vestingmuur, km blok 61-28-32, coord. 176.2/317.2, sur un mur en briques, 10.V.1998, P. Diederich 13652 (herb. P. Diederich).Romania, Dobrogea, Distr. Tulcea, in valle rivi Tirgusor, prope pagum Tirgusor, $50 \mathrm{~m}$, 12.VII.1973, A. Vězda. (MARSSJ, A.Vězda: Lichenes selecti exsiccati 1298). On L. versicolor.Slovkia, Súlovské skaly, Strážovské vrchy NE von Považska Bystrica, kalkreiche Konglomeratfelsen N vom Dorf Súlov, 400-600 m, 19.IV.1986, J. Poelt (GZU).- Spain: Aragón, prov. Zaragoza, Escatrón, Mocatero, U.T.M. 30TYL27-YL37, 190 m, 17.X.1991, P. NavarroRosinés (BC). On L. versicolor.- Prov. Zaragoza, am Nordufer des Embalse de Mequinensa N von Caspe, c. 200 m, Triften mit Kalksandsteinblöcken, 25.V.1983, J. Poelt (GZU).- Cantabria: Invernales de Liébana, calizas expuestas, J. Etayo (herb. J. Etayo). On L. versicolor.- Castilla-La Mancha: prov. Cuenca, Talayuelas, Pico Ranera, U.T.M. 30SXK4408, arenisca, 1400 m, 8.XI.1991, V. Calatayud (VAB-lich. 7446). On Lecanora bolcana.- Castilla y León: prov. Avila, Sierra de Gredos, cerca de Zapardiel de la Ribera, sobre granito, 1000 m, 16.III.1997, V. Calatayud (VABlich. 7765). On L. bolcana.- Catalunya: prov. Girona, Alt Empordà, el Port de la Selva, Salt de la Gorga (Cap de Creus), U.T.M. 31TEG1883, 150 m, 4.I.1985, X. Llimona (BCN-lich. 5546).Comunitat Valenciana: prov. Castelló, Azuebar, Bco. Mosquera, U.T.M. 30SYK2519, arenisca, 660 m, 26.X.1990, V. Calatayud (VAB-lich. 7445).- Prov. Castelló, Toro, Bco. Rasinero, U.T.M. 30SXK9122, arenisca, 880 m, 16.V.1992, V. Calatayud (VAB-lich. 7444 and herb. P. Diederich).Andalucía: Prov. Almería, Sierra Alhamilla NE von Almería, Trockenhänge etwa $4 \mathrm{~km} \mathrm{~S}$ von Lucainena de las Torres, N von Níjar, alt. ca. 600 m, 7.IV.1979, A. Buschardt (GZU).- See also 

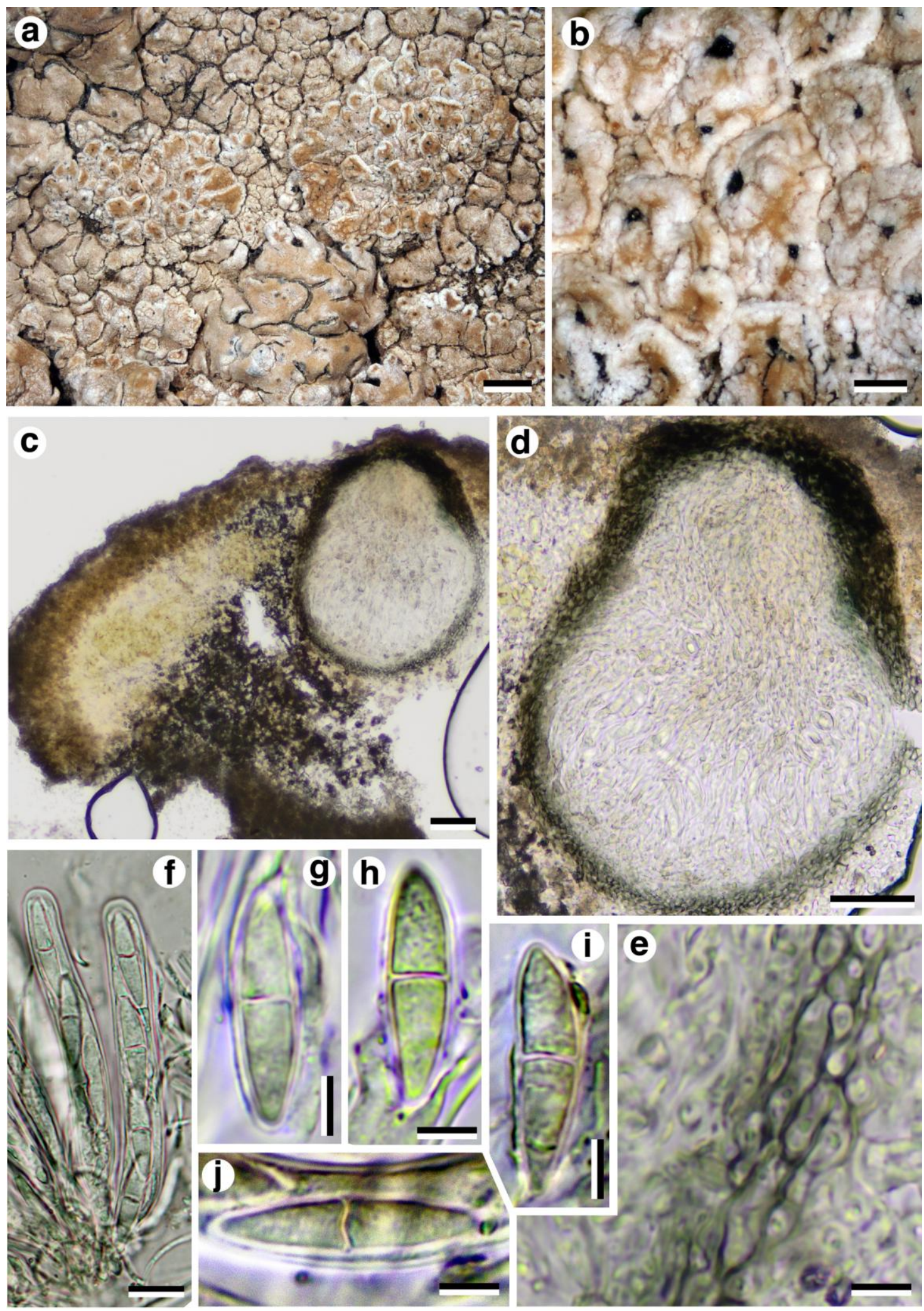

Fig 8-a-j, Cercidospora macrospora (Escatrón, Aragón, Spain). a, superficial aspect of parasitized thallus of Lecanora versicolor, with deformations induced by the fungus in the form of convex cecidia. $\mathbf{b}$, Detail of the superficial appearance of the fungal ascomata immersed in the lichen thallus. c, vertical section with a fungal ascoma immersed in the lichen thallus. d, detail of the ascoma in vertical section. e, detail of the exciple in vertical section from the lateral part of the ascoma. f, asci. g-j, ascospores. Bars: $\mathbf{a}=1 \mathrm{~mm} ; \mathbf{b}=0.2 \mathrm{~mm} ; \mathbf{c}, \mathbf{d}=50 \mu \mathrm{m} ; \mathbf{f}=10 \mu \mathrm{m} ; \mathbf{e}, \mathbf{g}$ $\mathbf{j}=5 \mu \mathrm{m}$ 
Hafellner (1987).- Sweden, Uppland, Österlövsta par., Lövstabruk ca. 5 km SE of Österlövsta, just $\mathrm{W}$ of the village, $16^{\circ} 24^{\prime} 30^{\prime}$ 'N $/ 17^{\circ} 57^{\prime} 30^{\prime}$ 'E, alt. ca. $25 \mathrm{~m}$; mixed forest with granitic outcrops; on old tiles beside the road, 11.V.1996, J. Hafellner (GZU).- United Kingdom: England, North Yorkshire, Cleveland, Ayton (near), Cliffrigg, sine data, W. Mudd (K 163704; Mudd, Lich. Brit. Exs. 287, possibly paratype material of Thelidium epipolytropum, at least from paratype locality).See also Hafellner (1987).-

ASIA: Afghanistan: Prov. Kabul, Paghman Gebirge, oberhalb des Ortes Paghman, an der Talgabelung Chap-Darrah und Rast-Darrah, 34³7'N/68'56'E, alt. $2550 \mathrm{~m}$, Silikatfelsen an der rechten Talseite, 21.VI.1970, M. Steiner Ste 59/13a (GZU). On L. dubyi.- Prov. Paktia, 10 km NE

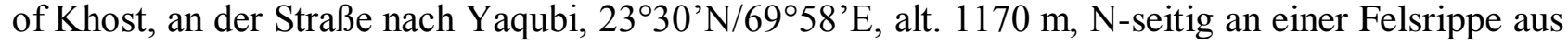
Vulkanit in der Schotterebene, 3.VII.1970, M. Steiner Ste 70/1a (GZU).- Japan, Hokkaido, ShaniSando, Esashi-gun, on cliffs along the coast, 17.VIII.1970, S. Kurokawa 70818a (separated from Kurokawa, Lich. Rar. Crit. exs. 225) (GZU).- Nepal, Central Himalaya, Langtang Area, Langshisa, 4100-4200 m, on big rocks, 15.IX.1986, J. Poelt N86-1733 (GZU, under the name of the host). Pakistan: Hindukush, S des Zani Passes, 2700 m, 3.VIII.1993, T. Peer (GZU, under the name of the host). On L. dubyi.- Baltistan, Northwestern Himalaya, eastern Deosai Plateau, $35^{\circ} 05^{\prime} \mathrm{N} / 75^{\circ} 34^{\prime} \mathrm{E}, 4100-4200 \mathrm{~m}$, rocky slope, 15.VII.1991, J. Poelt (GZU). On L. dubyi.- Baltistan, Karakorum, Haramosh Range, Chogo Lungma Valley, Gon E of Arandu, 35 $53^{\circ} \mathrm{N} / 75^{\circ} 22^{\prime}$ E, 30003200 m, 9.VIII.1991, J. Poelt (GZU). On L. dubyi.- Karakorum, Upper Kaghan Valley, Saiphul,

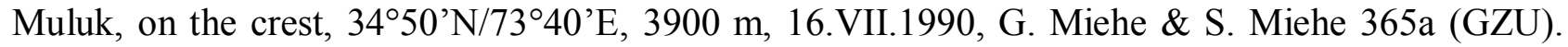
On L. dubyi.- Northwestern Himalaya, Indus Valley, rockery ca. $2 \mathrm{~km} \mathrm{~W}$ of Chilas, alt. c. $1300 \mathrm{~m}$, 21.VII.1991, J. Poelt K91-805 (GZU).- Russia: Yakutia, Mosmkii region, along the river Indigirka, c. $6 \mathrm{~km}$ SE of Tyubelyakh, $65^{\circ} 19^{\prime} \mathrm{N} / 143^{\circ} 15^{\prime} \mathrm{E}, 800 \mathrm{~m}, 16 . \mathrm{VII} .1992$, M.P. Zhurbenko 92297 (herb. P. Diederich). On terricolous "Placolecanora" sp.-

AfricA: Canary Isles: Tenerife, Las Cañadas, Boca de Tauce, northwest base of El Sombrerito, alt. 2000 m, 28.I.1964, H. A. Imshaug 34954a (GZU).- Tenerife, Las Cañadas, ca. 0,5 km W der Grenze des Nationalparks W ober El Portillo, 28 $17^{\prime} 10^{\prime}$ 'N/16 $34^{\circ} 10^{\prime}$ 'W, c. 2100 m, Felsköpfe aus vulkanischem Gestein, 20.II.1989, J. Hafellner 32868 \& A. Hafellner (GZU).Tenerife, Cumbre Dorsal, Izaña, sanft SE-geneigte Hänge zwischen dem Observatorio Meterológico und dem Observatorio Astronómico, 28¹8'10' N/16³0'15' 'W, c. 2350 m, kleine Vulkanitschrofen in einem Spartocytisus supranubius-Bestand, NE-seitig auf kleinen Vulkanitschrofen, 28.IV.2005, J. Hafellner 64313 (GZU).- Gran Canaria: N-Abhänge des Roque

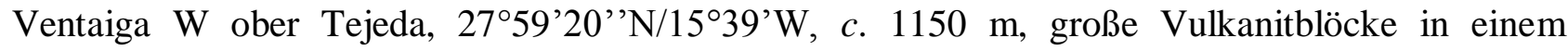
Mandelbaumhain, auf zeitweise besonnten Neigungsflächen, 22.II.1994, J. Hafellner 32858 (GZU). On L. garovaglii.- Morocco: Anti-Atlas, Tizi Aferni $1600 \mathrm{~m}$, sur quartzites, 8.V.1935, R.G. Werner (BC, herb. Werner). On L. garovaglii.- Bled Touloum dans les Ida n'Zal, sur calcaire, 8.V.1935, R.G. Werner (BC, herb. Werner). On L. versicolor.- Monts Zaians bled( ?) Teld, Ass A., terricole entre les pierres mobiles, 5.VI.1931, J. Gattefossé (BC, herb. Werner). On L. versicolor (kept at Werner's herbarium, and labeled as Squamarina periculosa).

North AMERICA: Canada, Alberta, E side of Alberta Hwy 6, 15.5 miles N of Twin Buttes, 2 miles S of Pincher Creek, $49^{\circ} 23^{\prime} \mathrm{N} / 113^{\circ} 55^{\prime} \mathrm{W}, 1220 \mathrm{~m}$, on exposed sandstone, 27.VII.1985, B. D. Ryan 14000a (GZU).- Ibid., B. D. Ryan 13939, 13948 (GZU). On L. garovaglii.- Mexico. See Hafellner (1987).- U.S.A: Arizona, Apache Co., along route 666 23,5 km N of St. Johns, shrubland

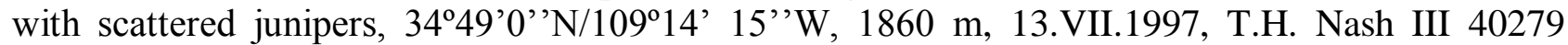
(ASU). On L. garovaglii.- Arizona, Apache Co., W end of Querino Wash, just S of Interstate Hwy

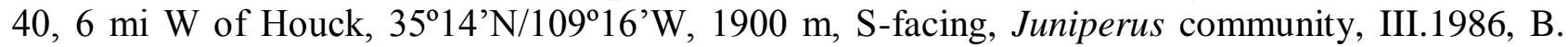
Ryan 19172 (ASU).- California, Kern Co., S side of California Hwy 178, 1 mile W of Onyx, 35'40'30''N/118'14'30' 'W, 915 m, 9.VI.1985, B. D. Ryan 15696 (GZU, under the name of the host). On L. dubyi.- Colorado, Garfield Co., E side of Hwy $13 \mathrm{~m}, 4.5 \mathrm{mi} \mathrm{N}$ of Rifle, $42^{\circ} 17^{\prime} \mathrm{N}$, $120^{\circ} 45^{\prime} 30^{\prime \prime} \mathrm{W}, 1700$ m., Pinyon-Junifer / Sagebrush community, S-facing hill, on sandstone, VIII. 1985, B. Ryan 20682 (ASU). On Lecanora novomexicana.- Michigan, Keweenaw Co., Isle Royale National Park, Pasage Island at NE tip on rock shore, 9.VII.1983, C. M. Wetmore 47525a (GZU).- 
Nevada, Lincoln Co., S end of Pahranagat Mts., along Hwy 93, ca. 16 miles S of Alamo, $37^{\circ} 15^{\prime} \mathrm{N} / 115^{\circ} \mathrm{W}$, elev. ca. 950 m, I.1986, B. D. Ryan 15738a (GZU).- Nevada, W of Las Vegas, Red Rock Canyon National Conservation Area, 1290 m, 3699'43' 'N/115'27'2''W, on noncalcareous sandstone rocks in an old quarry, 23.XII.1997, P. Diederich 14167 (herb. P. Diederich).Nevada, Washoe Co., E side of Pyramid Lake, road to the "Pyramid", 3956-57'N/119'25-27'W, 25.VI.1985, B.D. Ryan (GZU). On L. cf. nevadensis.- New Mexico, San Juan, G1, $36^{\circ} 50^{\prime} \mathrm{N} / 109^{\circ} 06^{\prime} \mathrm{W}, 6000^{\prime}$, on sandstone, 4.VII.1974, J. Marsh \& D. Rankert (ASU).- New Mexico, Santa Fe Co., $19 \mathrm{~km}$ NNW of White Lakes along US 285, 35 $22^{\prime} \mathrm{N} / 105^{\circ} 51^{\prime} \mathrm{W}$, alt. $1890 \mathrm{~m}$, on sandstone, 14.X.1983, T.H. Nash 22428a (GZU). On L. novomexicana.- Oregon, Lake Co., 5 mi E of Quartz Mountain, Drews Creek, Oregon Hwy 140, 42¹7'N/12045'30'’W, 1585 m., pine forest, on basalt, 28.VI.1985, B. Ryan 13856 (ASU). On L. phaedrophthalma var. christoi.

Cercidospora melanophthalmae Nav.-Ros., Calat. \& Hafellner, sp. nov.

Fig. 6, 9 f-1. MycoBank 516549

Ascomata perithecioidea, totaliter immersa in thallis aut apotheciis hospitis. In sectione transversali pseudothecia globosa, $170-330 \mu \mathrm{m}$ in diametro. Paries ascomatum apicaliter viridulocaerulescens, in externa parte leviter fusco-violascens, parce incrassatus, basaliter subhyalinus, circa $10-15 \mu \mathrm{m}$ crassus. Paraphysoides copiosae, $1-1.5(-2) \mu \mathrm{m}$ crassae. Asci cylindrici vel subclavati, (55-)70-90(-100) $\mu \mathrm{m}$ longi et 10-15 $\mu \mathrm{m}$ lati, octospori, rare hexaspori aut quadrispori. Ascosporae (16-)18-22(-24) $\times(4-) 5-6.5(-7) \mu \mathrm{m}$ magnae, incoloratae, ellipsoidei-fusiformes, ad septum non aut parum constrictae, cellula superiore breviore crassioreque quam cellula inferior, tenuiter halonatae. Cercidosporae epipolytropae affinis, sed ei dissimilis pseudotheciis et ascosporis majoribus. Supra thallis Rhizoplacae melanophthalmae vigens.

Type - Spain, Catalonia, prov. Girona, Baixa Cerdanya, Meranges, prop del refugi de Malniu, U.T.M.31TCH9902-DH0002, 2200 m, 13.X.1991, X. Llimona \& J.M. Pérez-Redondo (BCN-lich., holotype).

Host species of the type - Rhizoplaca melanophthalma (Ramond) Leuckert \& Poelt

Etymology - The specific epithet refers to the host species, Rhizoplaca melanophthalma.

Description - Ascomata perithecioid, 170-330 $\mu \mathrm{m}$ in diam., exciple blue-green in its upper part, sometimes violaceous brown in the outermost part, colorless towards its base, $10-15 \mu \mathrm{m}$ thick in this part. Paraphysoids abundant, 1-1.5(-2) $\mu \mathrm{m}$ thick. Asci $(55-) 70-90(-100) \times 10-15 \mu \mathrm{m}$, cylindrical-clavate, with (4-6-)8 spores. Ascospores (16-)18-19.7-22(-24) × (4-)5-5.7-6.5(7) $\mu \mathrm{m}$ with a length/width ratio of $(2.4-) 2.9-3.5-4.2(-4.8)(n=149)$, 1-septate, rarely simple, colorless, mostly ellipsoid-fusiform and attenuated at both extremes, less frequently oval-ellipsoid and only attenuated at one of the apices, slightly heteropolar, not or slightly constricted at the septum, guttulate.

Remarks - The occurrence of specimens of Cercidospora on Rhizoplaca melanophthalma has already been mentioned by other authors (Werner 1937, Navarro-Rosinés \& Hladun 1987, Alstrup \& Hawksworth 1990), who included them in a wider concept of C. epipolytropa. C. melanophthalmae and C. epipolytropa are the only two species in this study characterized by having predominantly 8-spored asci. Mature asci with only six or four ascospores may occur but are always rare among typical ones and contain usually aborted additional spores with smashed walls. Both taxa differ in the size of their ascomata, asci and ascospores, which are larger in $C$. melanophthalmae. The shape of the ascospores is also somewhat different; while in $C$. melanophthalmae they are mostly ellipsoid-fusiform, in C. epipolytropa they are oval-ellipsoid, with the upper apex rounded and the lower one slightly attenuated.

Other than Cercidospora epipolytropa, C. melanophthalmae does not show a preference to develop the ascomata in the apothecial discs. Ascomata are equally frequent in host thallus and apothecia but are more conspicuous in the later.

Distribution and habitat-Cercidospora melanophthalmae seems to be a specific parasymbiont of Rhizoplaca melanophthalma, and is known with certainty from the localities in the 
mountains of southern Norway, the French Alps, the French and Spanish Pyrenees, Corsica, the Caucasus, southern Syria, the Pamir-Karakorum-Himalaya mountain system, the Canary Islands, and Greenland reported in this study. To these should be added the citations of Cercidospora epipolytropa or one of its synonyms on R. melanophthalma by Vouaux (1913) and Rondon (1969) from the Western Alps in France, Lettau (1919) from Switzerland, Navarro-Rosinés \& Hladun (1987) from Catalonia (Spain), Vondrák \& Etayo (2007) from the Spanish Pyrenees, Lopez de Silanes et al. (1998) from northern Spain, Werner $(1937,1975)$ and Degelius (1966) from the Sierra Nevada (Andalucía, Spain), Werner (1934) from the Anti-Atlas in Morocco, as well as by Alstrup \& Hawksworth (1990) and Alstrup et al. (2009) from Greenland. The material cited by Werner (1937) could not be found in the herbarium of this author (BC, herb. R.-G. Werner). Alstrup \& Cole (1998) cite Cercidospora ulothii upon Rhizoplaca melanophthalma from the Canadian province British Columbia, as does Halıcı \& Aksoy (2009) from Turkey, collections which also might belong here.

Exsiccata - None.

Additional specimens examined - (All on Rhizoplaca melanophthalma).

EuroPE: France: Pyrénées-Orientales, SE de Eyne, 32.VII.1985, P. Diederich (herb. Diederich 6628).- Alpes septentrionales du Dauphiné, région du col de Lautaret, dalles horizontales et \pm inclinées de schistes gréseux, versant $S$ de la montagne de Chaillol, $\pm 2300 \mathrm{~m}$, VIII. 1957, G. Clauzade (MARSSJ, Lichenes Alpium 49).- Hautes Alpes, sommet de la butte, située à $300 \mathrm{~m}$ à l'E du col de Lautaret, 2089 m, 30.VIII.1955, G. Clauzade (MARSSJ, herb. B. de Lesdain).- Hautes Alpes, Lautaret, 10.VIII.1903, M. B. de Lesdain (MARSSJ, herb. M. Vouaux, sub Parcidia exiguella Nyl (ex herb. F. Camus dans l'herb A. Boistel).- Corse, mont Renoso, gros bloc rocheux granitique dans la vallée des Pozzi, 1800 m, 18.VIII.1970, R. Deschatres (BC, herb. Werner).Norway: Oppland, Lom: Jotunheimen, Visdalen, W-exponierte Hänge ca. $1 \mathrm{~km}$ NE von Spiterstulen, ca. $1250 \mathrm{~m}$, W-exponierte Abbrüche, 24.VIII.1984, J. Hafellner 12767 \& A. Ochsenhofer (herb. Hafellner).- Spain: Catalonia, prov. Girona, Ripollés, Queralbs, Vall de Núria, sota el Pic de l'Aliga, UTM 31TDG39, 2350 m, 1.VIII.1984, P. Navarro-Rosinés (BCN.-lich.).Ibid., Núria N von Ribes de Freser, NE von der Bergstation der Zahnradbahn, ca. 2100-2200 m, Südhänge mit subalpinen Rasen und Kalkschieferschrofen, 27.V.1983, J. Hafellner 17653 (GZU).Ibid., Núria N von Ribes de Freser, NW-Hänge SE über der Bergstation der Zahnradbahn, 2300 2400 m, Rücken und Schutt aus Kieselkalk und Kalkschiefer, 27.V.1983, J. Hafellner 17593 (herb. Hafellner).

ASIA: Afghanistan: Großer Pamir, Issiktal, ca. 4900 m s. m., auf einem Felsgrat, 3.VIII.1975, H. Huss 56 (GZU, under the name of the host).- Prov. Kabul, Tal des Pagman-Flusses, ca. $4 \mathrm{~km}$

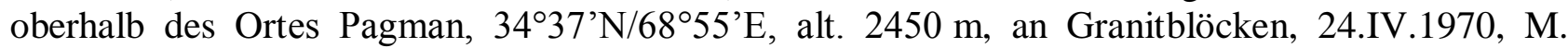
Steiner Ste 2a (GZU).- Prov. Baghlan, oberes Khinjan-Tal, bei der Brücke über den Khinjan-Fluss, $35^{\circ} 25^{\prime} \mathrm{N} / 69^{\circ} 00^{\prime} \mathrm{E}$, alt. $2600 \mathrm{~m}$, an Silikatblöcken am Fusse eines Steppenhanges mit Juniperus semiglobosa, 4.VI.1970,. M. Steiner Ste 40/8a (GZU).- Central Afghanistan, Deh Kundi, in summo jugo Khonak, alt. 3300 m, 1.VIII.1967, K. H. Rechinger (GZU).- Armenia: distr. Razdan: Caucasus, in declivibus occidentalibus montis Alibeg, in vicinitate pagi Cakhkdzor, alt. 18002000 m, 3.VII.1982, V. Vašak (GZU).- Pakistan: North Pakistan, Karakorum, on the pass Shinghai Gali, gentle ridge on the pass, $38^{\circ} 48^{\prime} \mathrm{N} / 74^{\circ} 10^{\prime} \mathrm{E}$, alt. $4470 \mathrm{~m}$, on small boulders, 28.VII.1990, G. Miehe \& S. Miehe 962a (GZU).- Karakorum, Basislager Jengutz Har (Spantik), alt. 4300 m, auf Schieferblöcken, 2.VII.1987, K. Cernic, herb. Hafellner 23221 (GZU).- NW Himalaya, Nanga Parbat-Gruppe, Diamirtal, Basislager, alt. 4600 m, Gneisfelsen, 3.VIII.1990, T. Peer (GZU).- North Pakistan, W Himalaya, Babusar Pass, $35^{\circ} 10^{\prime} \mathrm{N} / 74^{\circ} \mathrm{E}$, alt. $4300 \mathrm{~m}$, relatively humid alpine Cyperaceae mats influenced by solifluction, on small boulders in scree, 20.VII.1990, G. Miehe \& S. Miehe 515 (GZU, under the name of the host).- Hindukush, Tirich Valley, alt. $4200 \mathrm{~m}$, on siliceous rock, 7.VIII.1993, T. Peer (GZU).- Syria: Südsyrien, beim Dorf Mushannaf am Ostfuß des Dschebel Arab, 1989, H. Pölzl (GZU, under the name of the host).

AfricA: Canary Islands: Tenerife, Parque Nacional de El Teide, Roques de García, rocas volcánicas, 2125 m, 23.IX.1993, V. Calatayud (VAB-lich. 7452).- Tenerife, Las Cañadas, ca. 0,5 

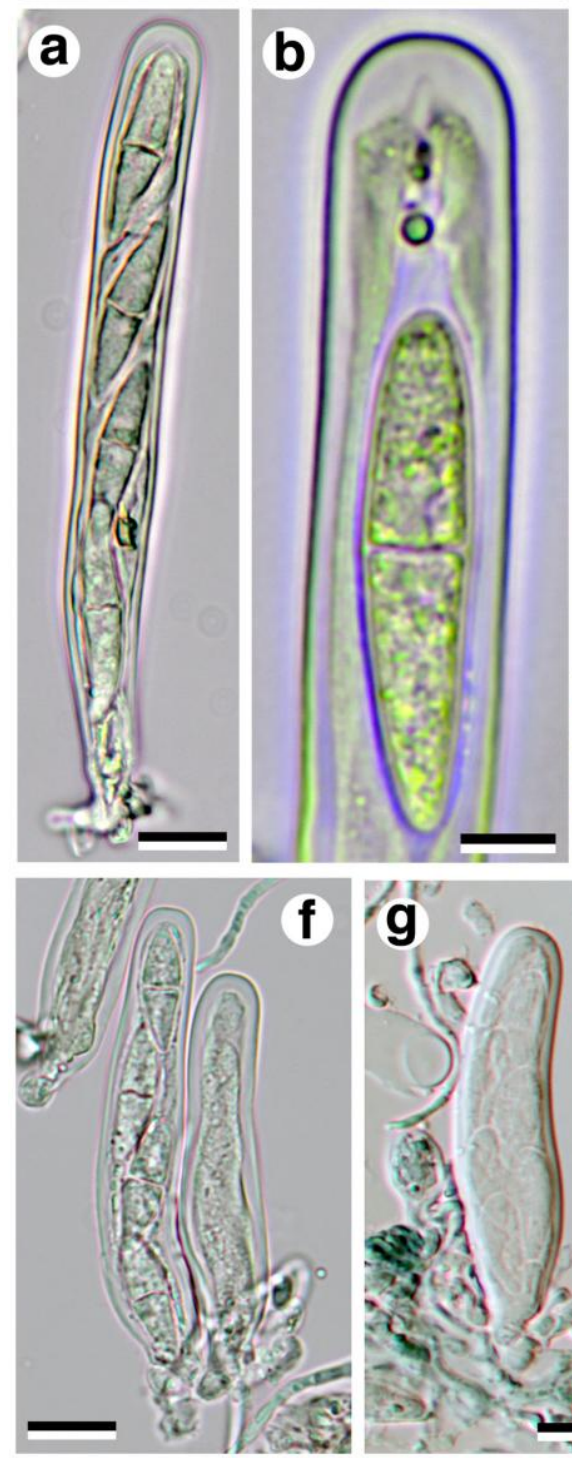
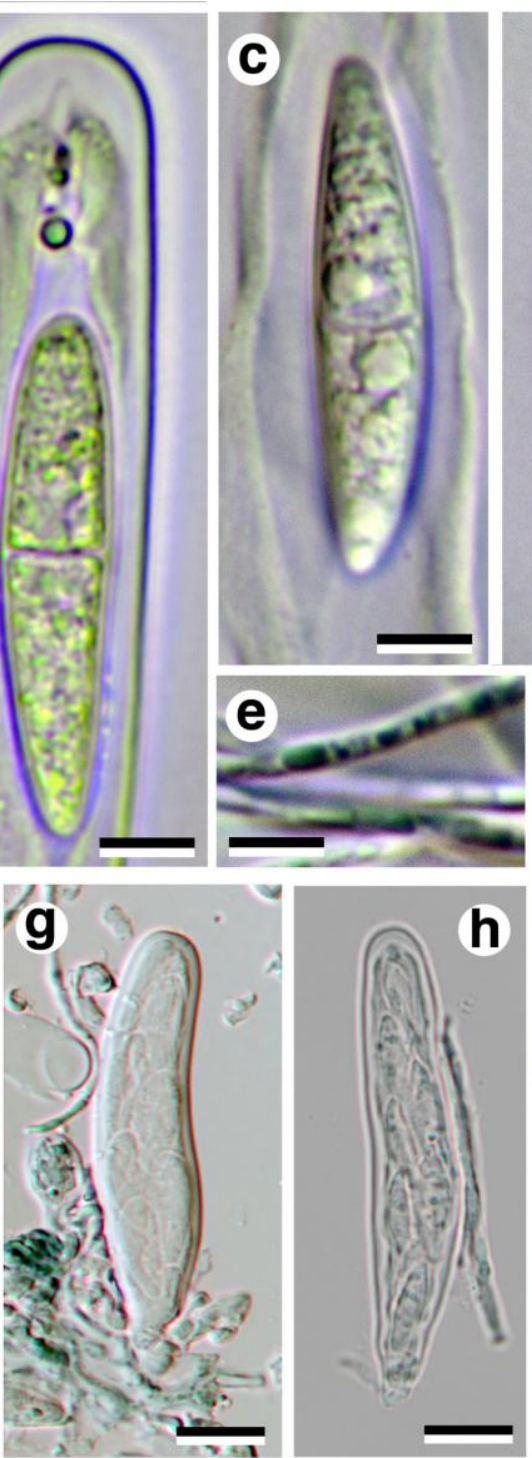
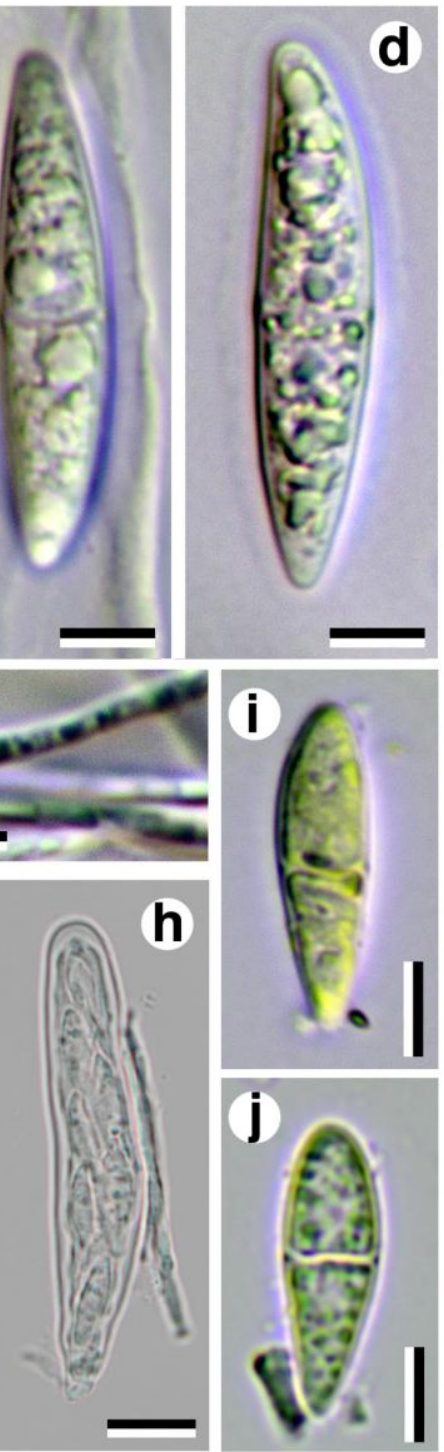
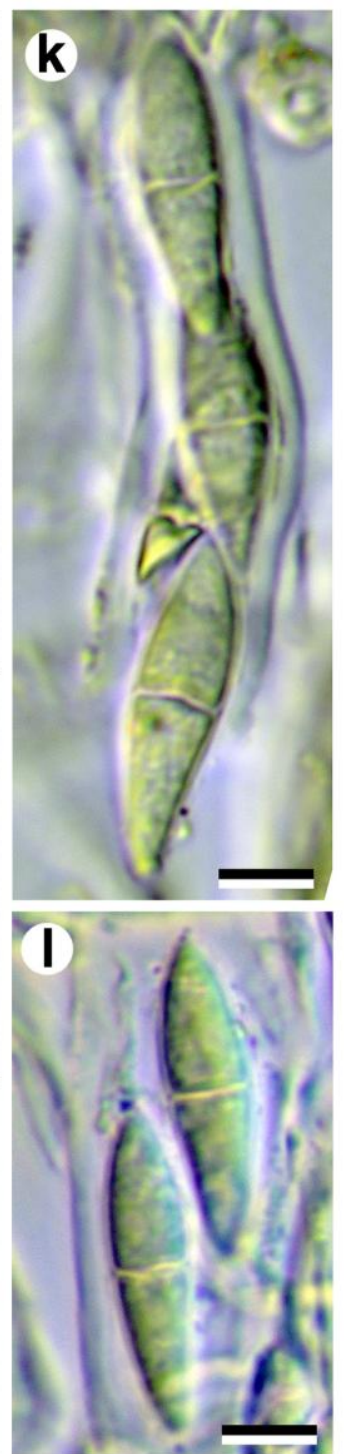

Fig. 9 - a-e Cercidospora barrenoana (Tenerife, Spain VAB 7436-holopype). a, ascus. b-d ascospores. e, paraphysoids. - f-1 Cercidospora melanophthalmae (Tenerife, Spain, VAB 7452). f-h immature asci. j-1 ascospores - Bars: $\mathrm{a}, \mathrm{f}-\mathrm{g}=10 \mu \mathrm{m}$; b-e, $\mathrm{i}-1=5 \mu \mathrm{m}$.

km W der Grenze des Nationalparks W ober El Portillo, 28 $8^{\circ} 17^{\prime} 10^{\prime}$ 'N/16 $34^{\prime} 10^{\prime \prime} \mathrm{W}$, ca. $2100 \mathrm{~m}$, Felsköpfe aus vulkanischem Gestein, 20.II.1989, J. Hafellner 41725 \& A. Hafellner (GZU).Tenerife, Las Cañadas, untere Abhänge der Guajara SE vom Parador, $28^{\circ} 13^{\prime} 00^{\prime}$ 'N $/ 16^{\circ} 37^{\prime} 25^{\prime}$ 'W, ca. $2200 \mathrm{~m}$, NW-exponierte schrofige Hänge mit offenem Spartocytisus supranubius-Gebüsch,NEexponiert an Vulkanitschrofen, 25.IV. 2005, J. Hafellner 64181 (herb. Hafellner).- Tenerife: Las Cañadas, Montaña Guajara, auf der S-Seite knapp unter dem Gipfel, 28 13 '00' 'N/16 36'40' 'W, ca. $2680 \mathrm{~m}$, E-seitig an Vulkanitblöcken, 21.II.1989, J. Hafellner 32862, 36214 \& A. Hafellner (GZU).- Tenerife, Las Cañadas, Sattel E unter der Guajara, 28 $12^{\prime} 30^{\prime}$ 'N $/ 16^{\circ} 35^{\prime} \mathrm{W}$, ca. $2250 \mathrm{~m}$, Gebirgshalbwüste, auf Lavablöcken, 8.XII.1997, J. Hafellner 41060 (GZU).- Tenerife: Las Cañadas, Montaña de las Arenas Negras SE oberhalb von El Portillo [gegen Montaña Colorada], $28^{\circ} 17^{\prime} 10^{\prime \prime} \mathrm{N} / 16^{\circ} 33^{\prime}$ 05' 'W, ca. $2250 \mathrm{~m}$, N-seitig an Basaltschrofen, 20.II.1989, J. Hafellner 36166 \& A. Hafellner (herb. Hafellner).- Tenerife, Cumbre Dorsal, Izaña, sanft SE-geneigte Hänge zwischen dem Observatorio Meterológico und dem Observatorio Astronómico, 28 18'10'N/ $16^{\circ} 30^{\prime} 15^{\prime}$ 'W, ca. $2350 \mathrm{~m}$, kleine Vulkanitschrofen in einem Spartocytisus supranubius-Bestand, NE-seitig auf kleinen Vulkanitschrofen, 28.IV. 2005, J. Hafellner 64331 (GZU). 
NorTH AMERICA: Greenland: W-Grönland, Disko, Lyngmark, untere Hänge des Lyngmarkjeld N Godhavn, Basalt 50-320 m, VII. 1983, J. Poelt \& H. Ullrich (GZU).- Disko, Umgebung von Godhavn, Gneisgebiet NE des Fjordes Kangerdlwarssuk, NE des Ortes Diskofjord, um 50 m, 4.VIII.1982, J. Poelt \& H. Ullrich (GZU).

Cercidospora stenotropae Nav.-Ros. \& Hafellner, ad int.

Fig. 4, 7 a-j.

Description - Ascomata perithecioid, (95-)110-150 $\mu \mathrm{m}$ diam., globose. Exciple colorless in its lower part, green-blue or with some brown tinges around the ostiole, $10-15 \mu \mathrm{m}$ thick towards its base. Paraphysoids abundant, 1-1.5 $\mu \mathrm{m}$ thick. Asci 40-55(-65) $\times 8-10(-12) \mu \mathrm{m}$, cylindricalclavate, with (2-)4 ascospores. Ascospores (13-)15-18.3-21(-22) $\times(4.5-) 5-5.3-6 \mu \mathrm{m}$, with a length/width ratio of (2.6-)3.0-3,5-4.0(-4.3) $(n=75)$, 1-septate, \pm narrowly ellipsoid to slightly fusiform, both cells with a similar size, septum median, but slightly heteropolar, the lower cell slightly narrower than the upper one. Pycnidia globose, 50-70 $\mu \mathrm{m}$ diam. Pycnidiospores colorless, simple, bacilliform, 3-5 × 0.5-1 $\mu \mathrm{m}$.

Remarks - Cercidospora stenotropae has the smallest ascomata and asci of all taxa herein treated. The asci are mostly 4-spored, but some 2-spored ones were also occasionally observed. Ascospore size is smaller than all 4-spored taxa and more closely resembles that found in $C$. melanophthalmae and $C$. epipolytropa, two species with mostly 8 -spored asci. In size and ellipsoidfusiform shape, $C$. stenotropae ascospores are almost identical to those of $C$. melanophthalmae. In C. epipolytropa they are slightly smaller and predominantly oval-ellipsoid (see also comments under these taxa).

Distribution and habitat $-C$. stenotropae is so far known with certainty from the localities here reported. This taxon grows in the apothecia of Lecanora stenotropa and other species of the $L$. polytropa group with scarcely developed thallus, in many cases reduced to only the margin of the apothecia. These lichen specimens always come from localities at lower altitudes (400-1600 m) than those where samples infected by $C$. epipolytropa were collected. It is quite probable that some reports of $C$. stenotropae have been included in a wide sense of $C$. epipolytropa.

A Cercidospora specimen from Antarctica growing on Rhizoplaca aspidophora, which was also examined, has similarly sized asci and ascospores with the same number of ascospores per ascus. Given the different host species, however, we prefer to list it separately under $C$. cf. stenotropae, until more material on this host is available for study.

Exsiccata - None.

Specimens examined - EUROPE: Austria: Steiermark, Cetische Alpen, Wechsel, LesesteinHaufen am Wiesenhang oberhalb des Spitzbauer, um 1000 m (an der Bergstraße von Mönichwald zum Wechsel), SE-exponiert, 8.IX.1989, J. Poelt (GZU). On L. polytropa group.- Fischbacher Alpen, Steiermark, Unterste N- bis W-seitige Abbrïche des Buchberges (N Schloß Herberstein) gegen den Stubenbergsee bzw. die Feistritz, 400-420 m, 6.XI.1988, J. Poelt (GZU). On L. polytropa group.- Belgium (distr. ardennais): Prov. Luxembourg, Arville, Sart-aux-Pîres, right side of the river Lomme IFBL: J6.56, $310 \mathrm{~m}$, on schistose rocks containing heavy metals on SW-exposed railway cutting, 4.V.1997, P. Diederich 12585 (herb. P. Diederich). On Lecanora gisleriana Müll. Arg.- Germany: Harz, Bez. Halle, Wald, Wegräder am Aufstieg von Drei-Annen-Hohne zu den Hohneklippen W Elbingerode, 800-900 m, 8.IX.1979, J. Poelt (GZU). On L. polytropa group.Sachsen-Anhalt, Eisleben-Kreisfeld, auf Kupferschiefer der Halde N vom Sportplatz Kreisfeld, 9.II.1994, leg. S. Huneck (herb. P. Diederich). On L. stenotropa.- Italy: Toscana, prov. Arezzo, Pratomagno W Bibbiena, Aufstieg von Raggiolo zum Kamm zwischen Croce di Pratomagno und Pgio. Masserecci, 1000 m, VII.1980, C. Scheuer (GZU). On L. polytropa group.- Spain: Castilla y León, prov. Soria, Molinos de Razón, Sierra Cebollera, alrededores de la laguna. sobre roca silicea, ca. 2000 m, 1.XI.1996, V. Calatayud \& C. Trescolí (VAB-lich. 7726). On L. polytropa group.Catalonia, prov. Girona, Baixa Cerdanya, Gréixer, cerca del pueblo U.T.M. 31TDG0396, 1400 m, 30.XI.1990, X. Llimona \& J.M. Pérez-Redondo (BCN-lich.). On L. stenotropa.- Catalonia, prov. 
Girona, Baixa Cerdanya, Meranges, prado cerca de Girult, U.T.M. 31TCH9900, 1600 m, 17.IV.1992, X. Llimona \& J.M. Pérez-Redondo (BCN-lich.). On L. stenotropa.

NoRTH AMERICA: U.S.A., Arizona: Apache Co., Mt. Baldy Wilderness, Baldy Peak, at the summit, ca. $3400 \mathrm{~m}, 33^{\circ} 54^{\prime} 30^{\prime}$ 'N/109'34'W; basaltic rock, on small outcrops exposed to SW, 4.VII.1994, J. Hafellner 36684 (GZU, under the name of the host). On L. polytropa.

Specimen examined of Cercidospora cf. stenotropae (on Rhizoplaca aspidophora).AnTARCTICA: Byero "El Petrel", cima rocosa, c. 15 m, 31.I.1990, L.G. Sancho (herb. J. Etayo 15117).

\section{Cercidospora sp. 1}

Remarks - This taxon, which was recently cited in the checklist of Virginia lichens, has not been available for study so far.

Distribution and habitat - This unknown Cercidospora has been recorded so far only from Virginia, U.S.A., where it was detected upon Rhizoplaca subdiscrepans (Hodkinson et al. 2009).

\section{Acknowledgements}

The authors are indebted to Dr. P. Diederich (Luxembourg) and Dr. C. Roux (Marseille) for their valuable comments on the manuscript. We thank the curators of the herbaria cited in the text for sending us material in their care for examination. P.N.-R.'s work was carried out within the projects: CGL 2007-66734-C03-02/BOS (Ministerio de Educación y Ciencia, Gobierno de España), 2005 SGR01047, GR-Criptogàmia (Departament d'Universitats, Recerca i Societat de la Informació, Generalitat de Catalunya). V.C. thanks Generalitat Valenciana, and the Programm CONSOLIDER-INGENIO 2010 (GRACCIE) for supporting to Fundación CEAM.

\section{References}

Alstrup V, Cole MS. 1998 - Lichenicolous fungi of British Columbia. Bryologist 101, 221-229.

Alstrup V, Hawksworth DL. 1990 - The lichenicolous fungi of Greenland. Meddel. Grønland. Biosci. 31, 1-90.

Alstrup V, Kocourková J, Kukwa M, Motiejūnaitė J, Brackel W, Suija A. 2009 - The lichens and lichenicolous fungi of South Greenland. Folia Cryptog. Estonica 46, 1-24.

Candal M, Halıc1 MG. 2011 - New Cercidospora records for Turkey. Turk. J. Bot. 35, 625-629.

Clauzade G, Roux C. 1985 - Likenoj de Okcidenta Eŭropo. Ilustrita determin-libro. Bull. Soc. Bot. Centre-Ouest, nouv. sér., Num. spéc. 7, 893 pp. Royan.

Degelius G. 1966 - Lichens of the summit of the Picacho de Veleta (Sierra Nevada, Spain). A contribution to the knowledge of the flora at high altitudes. Svensk Bot. Tidskr. 60(2), 338340 .

Grube M, Hafellner J. 1990 - Studien an flechtenbewohnenden Pilzen der Sammelgattumg Didymella (Ascomycetes, Dothideales). Nova Hedwigia 51(3-4), 283-360.

Grube M, Baloch E, Arup U. 2004 - A phylogenetic study of Lecanora rupicola group (Lecanoraceae, Ascomycota). Mycol. Res. 108(5), 506-514.

Hafellner J. 1987 - Studien über lichenicole Pilzen und Flechten VI. Ein verändertes Gattungskonzept für Cercidospora. Herzogia 7, 355-365.

Hafellner J, Casares-Porcel M. 2003 - Lichenicolous fungi invading lichens on gypsum soils in southern Spain. Herzogia 16, 123-133.

Halıcı MG, Aksoy A. 2009 - Lichenised and lichenicolous fungi of Aladağlar National Park (Niğde, Kayseri and Adana Provinces) in Turkey. Turkish J. Bot. 33, 169-189.

Hawksworth DL, Diederich P. 1988 - A synopsis of the genus Polycoccum (Dothideales), with a key to accepted species. Trans. Brit. Mycol. Soc. 90(2), 293-312.

Hodkinson BP, Harris RC, Case MA. 2009 - A checklist of Virginia lichens. Evansia 26(2), 64-88. 
Laundon JR. 2010 - Lecanora antiqua, a new saxicolous species from Great Britain, and the nomenclature and authorship of L. albescens, L. conferta and L. muralis. - Lichenologist 42(6), 631-636.

Lettau G. 1919 - Schweizer Flechten. II. Hedwigia 60(4), 267-312.

Lopez de Silanes ME, Terrón A, Etayo J. 1998 - Líquenes y hongos liquenícolas de Fuentes Carrionas, Sierra de Riaño y Valle de Liébana (N de España). Nova Acta Ci. Compostelana (Biol.) 8, 47-89.

Mudd W. 1861 - A manual of British lichens, containing description of all the species and varieties and five plates, with figures of the spores of one hundred and thirty species, illustrative of the genera. Darlington, For the author by Harrison Penney. 309 pp.

Miadlikowska J, Kauff F, Hofstetter V, Fraker E, Grube M, Hafellner J, Reeb V, Hodkinson BP, Kukwa M, Lücking R, Hestmark G, Garcia Otalora M, Rauhut A, Büdel B, Scheidegger C, Timdal E, Stenroos S, Brodo I, Perlmutter G, Ertz D, Diederich P, Lendemer JC, May P, Schoch CL, Arnold AE, Gueidan C, Tripp E, Yahr R, Robertson C, Lutzoni F. 2006 - New insights into classification and evolution of the Lecanoromycetes (Pezizomycotina, Ascomycota) from phylogenetic analyses of three ribosomal RNA- and two protein-coding genes. Mycologia 98, 1088-1103.

Navarro-Rosinés P, Hladun NL. 1987 - Aportación a los hongos liquenícolas, liquenizados o no, en Catalunya. Act. VI Simposio Nat. Bot. Cript., 431-440. Granada.

Navarro-Rosinés P, Roux C, Casares M. 1995 - Hongos liquenícolas de Squamarina, II, Sobre la identidad de "Didymella" crozalsiana (Ascomycetes). Cryptog., Bryol. Lichénol. 16(2), 99103.

Navarro-Rosinés P, Calatayud V, Hafellner J. 2004 - Cercidospora. pp. 635-639 in: Nash TH III, Ryan BD, Diederich P, Gries C, Bungartz F (eds.): Lichen Flora of the Greater Sonoran Desert Region, Volume 2. Lichens Unlimited, Arizona State University, Tempe, Arizona.

Navarro-Rosinés P, Calatayud V, Hafellner J. 2009 - Contributions to a revision of the genus Cercidospora (Dothideales) 1. Species on Megasporaceae. Mycotaxon 110, 5-25.

Obermayer W, Kantvilas G. 2003 - The identity of the lichens Siphula himalayensis and Lecanora teretiuscula. Herzogia 16, 27-34.

Pérez-Ortega S, Spribille T, Palice Z, Elix JA, Printzen C. 2010 - A molecular phylogeny of the Lecanora varia group, including a new species from western North America. Mycol. Progress 9, 523-535.

Poelt J, Grube M. 1993 - Beiträge zur Kenntnis der Flechtenflora des Himalaya VIII. - Lecanora subgen. Placodium. Nova Hedwigia 57, 305-352.

Purvis OW, Coppins BJ, Hawksworth DL, James PW, Moore DM. 1992 - The lichen flora of Great Britain and Ireland. London: Natural History Museum Publications and the British lichen Society. $710 \mathrm{pp}$.

Rondon Y. 1969 - L'herbier des champignons parasites des lichens de l'abbé L. Vouaux. Rev. Bryol. Lichénol. 36, 737-745.

Santesson R. 1960 - Lichenicolous fungi from northern Spain. Svensk Bot. Tidskr. 54, 499-522.

Santesson R. 1993 - The lichens and lichenicolous fungi of Sweden and Norway. Lund: SBTförlaget. $240 \mathrm{pp}$.

Santesson, R. 1998 - Fungi lichenicoli exsiccati. Fasc. 11 \& 12 (Nos 251-300). Thunbergia 28, 119.

Triebel D, Rambold G, Nash III TH. 1991 - On lichenicolous fungi from continental North America. Mycotaxon 42, 263-296.

Vainio EA. 1921 - Lichenografia fennica I. Pyrenolichenes iisque proximi Pyrenomycetes et Lichenes imperfecti. Acta Soc. Fauna Fl. Fenn. 49(2), 1-274.

Vondrák J, Etayo J. 2007 - A contribution to the diversity of lichen-forming and lichenicolous fungi in the Spanish Pyrenees. Herzogia 20, 189-198.

Vouaux L. 1912-1914 - Synopsis des champignons parasites de liquens. Bull. Soc. Mycol. France 28(1912), 177-256; 29(1913), 33-128, 395-494; 30(1914), 135-198, 281-329. 
Werner R-G. 1934 - Étude sur la végétation cryptogamique du massif du Siroua (Anti-Atlas). Bull. Soc. Sci. Nat. Maroc 14, 214-235.

Werner R-G. 1937 - Recherches phytogéographiques comparées sur la flore cryptogamique de l'Espagne méridionale et du Maroc. Bull. Soc. Sci. Nat. Maroc 17, 32-66.

Werner R-G. 1975 - Étude écologique et phytogéographique sur les lichens de l'Espagne méridionale. Rev. Bryol. Lichénol. 41, 55-82.

Wirth V. 1995 - Flechtenflora. 2. Auflage. Stuttgart, E. Ulmer. 661 pp.

Zhurbenko MP. 2007 - The lichenicolous fungi of Russia, geographical overview and a first checklist. Mycologia Balcanica 4(3), 105-124. 